\title{
Multiple critical points of saddle geometry functionals
}

\author{
Jonáš Volek*1 \\ ${ }^{1}$ NTIS - New Technologies for the Information Society (research center), \\ Faculty of Applied Sciences, University of West Bohemia, \\ Univerzitní 8, 30100 Pilsen, Czech Republic
}

\begin{abstract}
We study the multiplicity of critical points for continuously differentiable functionals on real Banach spaces. We prove that a functional which satisfies the assumptions of the Saddle Point Theorem and moreover is bounded from below has at least three critical points. Apparently, there is a global minimizer and a saddle point and we show the existence of a third critical point. The idea of the proof is based on the minus-gradient flow. This result is closely related to the three critical points theorem of H. Brezis and L. Nirenberg which assumes a local linking. Finally, we apply the result on the Dirichlet problem for semilinear stationary PDEs. The analysis includes, for example, the existence of multiple stationary solutions of bistable (or Allen-Cahn) equation and semipositone problems.
\end{abstract}

Keywords: critical point theory; multiplicity; saddle geometry; Saddle Point Theorem; boundedness from below; stationary PDEs.

MSC2010: 49J27; 49J35; 58E05; 35A15.

\section{Introduction and main result}

The critical point theory has proved to be a very important and efficient tool in mathematics. It readily became one of fundamental building blocks of nonlinear analysis. Especially, the connection with the existence theory for differential equations has attracted a lot of attention. Now it is a standard way of proving miscellaneous results such as bifurcation theorems, mentioned existence results for differential equations, stability results in the theory of dynamical systems and many others (see, e.g., J. Mawhin, M. Willem [24]).

We study the multiplicity of critical points for continuously differentiable functionals on real Banach spaces which has certain saddle-type geometry. In 1978 P. H. Rabinowitz [32] introduced the Saddle Point Theorem which became quickly an essential part of the critical point theory and one of principal minimax theorems. It relies on a special geometrical assumption which naturally abstracts the idea of saddle points in finite dimension. In this paper we show that if the considered functional satisfies the P. H. Rabinowitz's saddle assumption (denoted below by $(R))$ together with the Palais-Smale compactness condition and additionally is bounded from below, it has at least three critical points.

The following theorem is our main result. We denote by $B_{Y}(R)$ the open ball with the radius $R>0$ in the subspace $Y \subset X$ centered at the origin, by $\partial B_{Y}(R)$ the corresponding sphere in $Y$ and by $X^{*}$ the dual space of $X$.

Theorem 1.1. Let $X$ be a real Banach space, $X=Y \oplus Z$ where $Y \neq\{o\}$ is finite dimensional. Assume that $F \in C^{1}(X, \mathbb{R})$, is bounded from below and satisfies

(R) there exists $R>0$ such that $\max _{u \in \partial B_{Y}(R)} F(u)<\inf _{u \in Z} F(u)$,

and the Palais-Smale condition

$(P S)$ every sequence $\left\{u_{n}\right\} \subset X$ such that $\left\{F\left(u_{n}\right)\right\} \subset \mathbb{R}$ is bounded and $\left\|F^{\prime}\left(u_{n}\right)\right\|_{X^{*}} \rightarrow 0$ possesses a convergent subsequence.

Then $F$ has at least three critical points.

The existence of two critical points in Thm. 1.1, specifically the global minimizer and a saddle point, follows immediately from one of minimization principles and from the Saddle Point Theorem. More ambitious problem is to show the presence of a third critical point.

Thm. 1.1 uses the splitting $X=Y \oplus Z$ where $Y$ is a finite dimensional subspace. For $\operatorname{dim} Y=1$ there exists a mountain range of $F$ formed along the subspace $Z$ separating $X$ into two parts. Consequently, there is a minimizer

*e-mail address: volek1@ntis.zcu.cz 


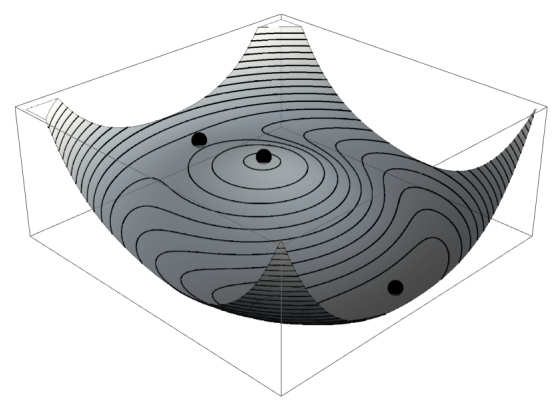

(a) The graph of function $F(\cdot, \cdot, 0)$ for $F$ given by (1.1) with its critical points.

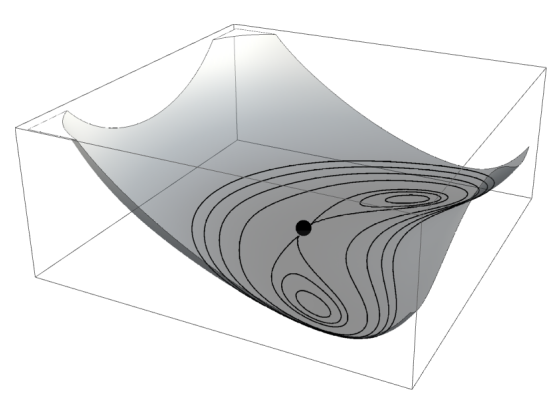

(b) The graph of function $F$ given by (1.2) illustrating a saddle which is not covered by the assumption (BN).

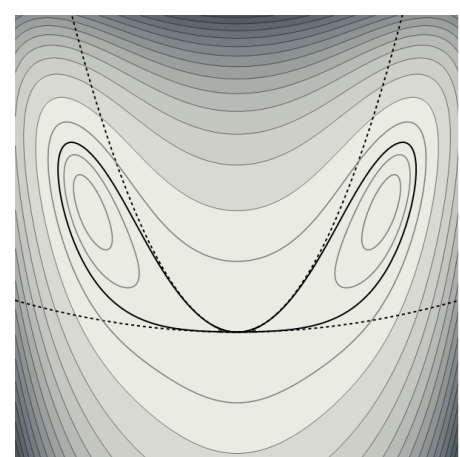

(c) Contours of function $F$ given by (1.2) with the zero contour being highlighted and squeezed by two parabolas, see (1.3).

Figure 1

on each side of that mountain range, since we assume that the functional is bounded from below. However, the subspace $Z$ does not have the separation property provided $\operatorname{dim} Y>1$. Hence, there need not be a second minimizer in this case.

As an example consider the function $F: \mathbb{R}^{3} \rightarrow \mathbb{R}$ defined by

$$
F(x, y, z)=\left(x^{2}+y^{2}\right)\left(x^{2}+y^{2}-1\right)+\frac{y}{4}+z^{2} .
$$

One can easily show that the function $F$ satisfies assumptions of Thm. 1.1 with $Y=\{(x, y, 0)\}$ and $Z=\{(0,0, z)\}$ for which $\operatorname{dim} Y=2$. Nonetheless, the function $F$ has a unique minimizer. More precisely, $F$ has the global minimizer and two saddle points (see Fig. 1a).

Consequently, we encounter a challenging aspect in the proof of existence of third critical point. We treat the case in which the dimension of subspace $Y$ in $X=Y \oplus Z$ is not necessarily equal to one and thus, $Z$ need not have the separation property.

There is a well-known result of $\mathrm{H}$. Brezis and L. Nirenberg which is very similar to Thm. 1.1. We formulate it in the following way (see H. Brezis, L. Nirenberg [6, Thm. 4]).

Theorem 1.2. Let $X$ be a real Banach space, $X=Y \oplus Z$ where $Y$ is finite dimensional. Assume that $F \in C^{1}(X, \mathbb{R})$, $F(o)=0$, is bounded from below with $\inf _{u \in X} F(u)<0$, satisfies

(BN) there exists $R>0$ such that

$$
\left\{\begin{array}{lll}
F(u) \leq 0 & \text { for } & u \in \bar{B}_{Y}(R), \\
F(u) \geq 0 & \text { for } & u \in \bar{B}_{Z}(R),
\end{array}\right.
$$

and the Palais-Smale condition (PS). Then $F$ has at least two nonzero critical points.

The difference between Thm. 1.1 and H. Brezis' and L. Nirenberg's Thm. 1.2 is in the saddle geometry assumptions $(R)$ and $(B N)$. The assumption $(B N)$ puts conditions on the local linking and is restricted only to the neighborhood of the origin which is supposed to be a critical point. Nevertheless, it controls the behavior of the corresponding functional on whole balls $\bar{B}_{Y}(R), \bar{B}_{Z}(R)$. Consequently, for example the function $F: \mathbb{R}^{2} \rightarrow \mathbb{R}$ defined by

$$
F(x, y)=\left(y-x^{2}\right)\left(y-2 x^{2}\right)+x^{6}+y^{6},
$$

does not satisfy $(B N)$ (see Fig. 1b). Note that $F(0,0)=0,(0,0) \in \mathbb{R}^{2}$ is a saddle point and the zero sublevel set

$$
F^{0}=\left\{(x, y) \in \mathbb{R}^{2}: F(x, y) \leq 0\right\},
$$

lies between two parabolas, specifically,

$$
F^{0} \subset\left\{(x, y) \in \mathbb{R}^{2}: x^{2} \leq y \leq 2 x^{2}\right\} .
$$

Hence, there is no splitting $X=\mathbb{R}^{2}=Y \oplus Z$ such that (BN) is satisfied (see Fig. 1c).

On the contrary, the assumption $(R)$ is not local and bounds the infimum of the functional over the whole subspace $Z$. However, it controls the behavior of the functional only on the sphere $\partial B_{Y}(R)$ and not on the whole ball $\bar{B}_{Y}(R)$ in the subspace $Y$. Consequently, the assumption $(R)$ involves other types of saddles (e.g., the function $F$ given by (1.2)). Moreover, in the case of Thm. 1.1 the initial saddle point guaranteed by the Saddle Point Theorem is not a priori localized at the origin. 
Besides this closest result of H. Brezis and L. Nirenberg, we want to emphasize another related references. G. Bonanno in $[4,5]$ studies a characterization of the mountain pass geometry introduced firstly in the Mountain Pass Theorem by A. Ambrosetti and P. H. Rabinowitz in [2] and which was lately generalized by P. Pucci and J. Serrin in [29] and by N. Ghoussoub and D. Preiss in [15]. He proves in [4] that the generalized mountain pass geometry is equivalent to the existence of two local minima provided the functional is bounded from below as in our case. The proof is based on the above mentioned separation of the underlying space into two parts by a mountain range which is formed along a sphere given by the mountain pass geometry. As an immediate consequence he obtains a three critical points theorem. In [5] the author studies a more general case of functionals bounded from below only on bounded subsets.

The literature about the critical point theory is wide and rapidly increases. There are many related references connected with uniqueness or multiplicity of critical points. Let us briefly mention some other relevant and extending papers to our issues. From the papers which deal with the uniqueness of critical points we emphasize the works of A. C. Lazer, E. M. Landesman, D. R. Meyers [19] and R. Manásevich [23] where the uniqueness of global saddle points is shown. Their results are based also on the splitting $X=Y \oplus Z$ and use concavity and convexity assumptions on the functional on these subspaces.

From the papers that deal with the multiplicity of critical points we want to highlight the following ones besides the above discussed works of H. Brezis, L. Nirenberg [6] and G. Bonanno [4, 5]. Let us mention the paper of P. H. Rabinowitz [30] which studies, using topological degree, whether the presence of two critical points implies the existence of a third one. This work was later followed by A. Castro and A. C. Lazer in [7]. They also use the splitting $X=Y \oplus Z$ and concavity and convexity assumptions. More recently, three critical points problems was studied by B. Ricceri in, e.g., [33], and four critical points theorems by Z. Liu and J. Sun [22].

Furthermore, there are many works that use symmetry assumptions, e.g., the evenness of the functional, to show that there exist multiple and possibly infinitely many critical points (see, e.g., P. H. Rabinowitz [31, Sec. 89]). As an example, one can mention the result of D. C. Clark from [10] which is based on the properties of the Ljusternik-Schnirelmann category. There are also several extensions of these results for perturbations of symmetric functionals (see, e.g., P. H. Rabinowitz [31, Sec. 10]).

Many authors follow the above listed results. For example, D. Kandilakis, N. C. Kourogenis, N. S. Papageorgiou [17] and X. Wu [34] generalize Thm. 1.2 of H. Brezis and L. Nirenberg for nonsmooth functionals. Further, $\mathrm{S}$. Li and M. Willem extend Thm. 1.2 in their paper [20] into the case in which the dimension of $Y$ in $X=Y \oplus Z$ is not finite.

Our paper is organized as follows. In Sec. 2 we sum up all needed fundamental results and tools. Further, in Sec. 3 we show a topological lemma, whose application is crucial in the proof of Thm. 1.1. Then in Sec. 4 we present the complete proof of Thm. 1.1 based on the idea of minus-gradient flow. In the last Sec. 5 we apply Thm. 1.1 on the Dirichlet boundary value problem for stationary semilinear PDEs which include, e.g., stationary problems for bistable (or Allen-Cahn) equation and semipositone problems.

\section{Preliminaries}

In this preliminary section we sum up fundamental results which are later needed in the proof of Thm. 1.1. The first statement is one of minimization principles which we apply to show the existence of the global minimizer of the functional $F$ in Thm. 1.1 (see, e.g., P. H. Rabinowitz [31, Thm. 2.7]).

Theorem 2.1. Let $X$ be a real Banach space. Assume that $F \in C^{1}(X, \mathbb{R})$, is bounded from below and satisfies $(P S)$. Then $c=\inf _{u \in X} F(u)$ is a critical value of $F$.

The second result is the Saddle Point Theorem due to P. H. Rabinowitz [32] which guarantees the existence of a second critical point of $F$ in Thm. 1.1 besides the global minimizer (see also the survey paper P. H. Rabinowitz [31, Thm. 4.6]).

Theorem 2.2. Let $X$ be a real Banach space, $X=Y \oplus Z$ where $Y \neq\{o\}$ is finite dimensional. Assume that $F \in C^{1}(X, \mathbb{R})$ and satisfies $(R)$ and $(P S)$. Then $F$ possesses a critical value $c \geq \inf _{u \in Z} F(u)$.

The idea of the proof of a third critical point of $F$ in Thm. 1.1 is based on the construction of a minus-gradient flow. However, the notion of gradient is not available, since we work on a Banach space. Despite this fact, we can use the so-called pseudo-gradient vector field introduced by R. S. Palais [28] who also proved the following statement about the existence of a pseudo-gradient for smooth functionals on Banach spaces (see also P. H. Rabinowitz [31, Lem. A.2]).

Theorem 2.3. Let $X$ be a real Banach space. If $F \in C^{1}(X, \mathbb{R})$, then there exists a pseudo-gradient vector field $v: X \backslash \Gamma \rightarrow X$ for $F$, where $\Gamma=\left\{u \in X: F^{\prime}(u)=o\right\}$, i.e., a locally Lipschitz mapping $v: X \backslash \Gamma \rightarrow X$ such that for all $u \in X \backslash \Gamma$ the following hold:

(i) $\|v(u)\|_{X} \leq 2\left\|F^{\prime}(u)\right\|_{X^{*}}$,

(ii) $\left\langle F^{\prime}(u), v(u)\right\rangle \geq\left\|F^{\prime}(u)\right\|_{X^{*}}^{2}$.

The last result presented in this section is a consequence of the Ekeland Variational Principle which provides the existence of a Palais-Smale subsequence (i.e., a subsequence satisfying the assumptions of the Palais-Smale condition) of every minimizing sequence of a functional which is bounded from below (see I. Ekeland [13, Thm. 2.2]). 
Theorem 2.4. Let $X$ be a real Banach space. Assume that $F: X \rightarrow \mathbb{R}$ is Fréchet differentiable, bounded from below and a sequence $\left\{u_{n}\right\} \subset X$ satisfies $F\left(u_{n}\right) \rightarrow \inf _{u \in X} F(u)$. Then there exists a sequence $\left\{\nu_{n}\right\} \subset X$ such that the following hold:

(i) $F\left(\nu_{n}\right) \leq F\left(u_{n}\right)$ for all $n \in \mathbb{N}$,

(ii) $\left\|u_{n}-\nu_{n}\right\|_{X} \rightarrow 0$,

(iii) $\left\|F^{\prime}\left(\nu_{n}\right)\right\|_{X^{*}} \rightarrow 0$.

\section{Topological lemma}

Besides the fundamental results from Sec. 2, we apply a topological argument as is usual in proofs of variational statements. We need the following lemma, which says in other words that a continuous homotopy in a finite dimensional space which deforms a sphere centered at the origin into a convex set separated from the origin has to cross the origin.

Lemma 3.1. Let $Y \neq\{o\}$ be a finite dimensional normed linear space. Assume that $h:[0,1] \times \partial B_{Y}(R) \rightarrow Y$ is a continuous mapping such that:

(i) $h(0, u)=u$ for all $u \in \partial B_{Y}(R)$,

(ii) there exists a convex set $K \subset Y$ such that $o \notin K$ and $h(1, u) \in K$ for all $u \in \partial B_{Y}(R)$.

Then there exist $t_{0} \in(0,1)$ and $u_{0} \in \partial B_{Y}(R)$ such that $h\left(t_{0}, u_{0}\right)=o$.

Proof. Let $w \in h\left(1, \partial B_{Y}(R)\right)$ be arbitrary and fixed. Construct a continuous homotopy $H:[0,2] \times \partial B_{Y}(R) \rightarrow Y$ as

$$
H(t, u)= \begin{cases}h(t, u), & t \in[0,1], \\ h(1, u)+(t-1)(w-h(1, u)), & t \in(1,2] .\end{cases}
$$

Furthermore, let $\pi: Y \backslash\{o\} \rightarrow \partial B_{Y}(R)$ be the radial projection of $Y \backslash\{o\}$ onto $\partial B_{Y}(R)$, i.e., $\pi(u)=\frac{R u}{\|u\|_{Y}}$. Obviously, $\pi$ is continuous on $Y \backslash\{o\}$.

Assume by contradiction that the statement of lemma does not hold, i.e., $h(t, u) \neq o$ for all $t \in[0,1]$ and all $u \in \partial B_{Y}(R)$. Then also

$$
H(t, u) \neq o \text { for all } t \in[0,2] \text { and } u \in \partial B_{Y}(R) .
$$

Indeed, for $t \in[0,1]$ there is $H(t, u)=h(t, u) \neq o$ for all $u \in \partial B_{Y}(R)$ via the assumption. For $t \in(1,2]$ there is

$$
H(t, u)=h(1, u)+(t-1)(w-h(1, u)),
$$

i.e., $H(t, u)$ is a convex combination of $w \in K$ and $h(1, u) \in K$. Since $K$ is a convex set and $o \notin K$ by the assumption, we obtain that $H(t, u) \neq o$ also for all $t \in(1,2]$ and $u \in \partial B_{Y}(R)$. Hence, (3.1) holds true.

Therefore, the mapping $\pi \circ H:[0,2] \times \partial B_{Y}(R) \rightarrow \partial B_{Y}(R)$ is well-defined and continuous. Moreover,

and

$$
\pi(H(0, u))=\pi(h(0, u))=\pi(u)=u \quad \text { for all } u \in \partial B_{Y}(R),
$$

$$
\pi(H(2, u))=\pi(w)=\frac{R w}{\|w\|_{Y}} \quad \text { for all } \quad u \in \partial B_{Y}(R) .
$$

This shows that the sphere $\partial B_{Y}(R)$ is contractible into the point $\frac{R w}{\|w\|_{Y}} \in \partial B_{Y}(R)$ (see, e.g., P. Drábek, J. Milota [12, Def. 5.1.8]). However, every finite dimensional sphere is not contractible (see, e.g., P. Drábek, J. Milota [12, Lem. 5.1.10]), a contradiction.

\section{Proof of Theorem 1.1}

After the brief summary of required tools, we present in this section the complete proof of Thm. 1.1 divided into several steps. Throughout the section we suppose that the assumptions of Thm. 1.1 are satisfied. We introduce the following notation for the brevity

$$
a=\max _{u \in \partial B_{Y}(R)} F(u), \quad b=\inf _{u \in Z} F(u) .
$$

Hence, there is $a<b$ by $(R)$.

Let us start with the following lemma about the existence of at least two critical points of the functional $F$. It is an immediate consequence of Thm. 2.1 and Thm. 2.2. 
Lemma 4.1. Let the assumptions of Thm. 1.1 be satisfied. Then there exist at least two critical points $u_{M}, u_{S} \in X$ of $F$ such that

$$
F\left(u_{M}\right)=\min _{u \in X} F(u), \quad F\left(u_{S}\right) \geq b .
$$

Particularly, there is $F\left(u_{M}\right)<F\left(u_{S}\right)$.

Denote by $\Gamma=\left\{u \in X: F^{\prime}(u)=o\right\}$ the set of all critical points of the functional $F$. We prove Thm. 1.1 by contradiction. Hence, we suppose that $u_{M}, u_{S}$ are the only critical points of $F$, i.e.,

$$
\Gamma=\left\{u_{M}, u_{S}\right\}
$$

Since $F \in C^{1}(X, \mathbb{R})$, Thm. 2.3 provides the existence of a pseudo-gradient vector field $v: X \backslash \Gamma \rightarrow X$ satisfying for all $u \in X \backslash \Gamma$

$$
\begin{gathered}
\|v(u)\|_{X} \leq 2\left\|F^{\prime}(u)\right\|_{X^{*}}, \\
\left\langle F^{\prime}(u), v(u)\right\rangle \geq\left\|F^{\prime}(u)\right\|_{X^{*}}^{2} .
\end{gathered}
$$

The continuity of $F$ and the inequalities $F\left(u_{S}\right) \geq b>a$ imply that there exists $\rho_{S}>0$ such that $F(u)>\frac{a+b}{2}>a$ for all $u \in \bar{B}\left(u_{S}, \rho_{S}\right)$, where $\bar{B}\left(u_{S}, \rho_{S}\right)$ denotes the closed ball in $X$ with the radius $\rho_{S}>0$ centered at $u_{S} \in X$. We define sets

$$
H=\bar{B}\left(u_{S}, \rho_{S}\right), \quad G=\bar{B}\left(u_{S}, \frac{\rho_{S}}{2}\right) .
$$

Similarly, for every $n \in \mathbb{N}$ there exists $\rho_{n}>0$ such that $\bar{B}\left(u_{M}, \rho_{n}\right) \cap H=\emptyset$ and $F(u)<F\left(u_{M}\right)+\frac{1}{n}$ for all $u \in \bar{B}\left(u_{M}, \rho_{n}\right)$. We define further the sets

$$
B_{n}=\bar{B}\left(u_{M}, \rho_{n}\right), \quad A_{n}=\bar{B}\left(u_{M}, \frac{\rho_{n}}{2}\right) .
$$

Let $n \in \mathbb{N}$ be given. Define mappings $h: X \rightarrow[0,1], f_{n}: X \rightarrow[0,1]$ as

$$
h(u)=\left\{\begin{array}{ll}
0, & u \in G, \\
\frac{2}{\rho_{S}}\left\|u-u_{S}\right\|_{X}-1, & u \in H \backslash G, \\
1, & u \in X \backslash H,
\end{array} \quad f_{n}(u)= \begin{cases}0, & u \in A_{n}, \\
\frac{2}{\rho_{n}}\left\|u-u_{M}\right\|_{X}-1, & u \in B_{n} \backslash A_{n}, \\
1, & u \in X \backslash B_{n} .\end{cases}\right.
$$

Furthermore, define $r:[0, \infty) \rightarrow(0,1]$ as

$$
r(s)= \begin{cases}1, & s \in[0,1] \\ \frac{1}{s}, & s \in(1, \infty)\end{cases}
$$

It is not hard to see that all the mappings $h, f_{n}, r$ are Lipschitz on their domains. Finally, we define the following operator $g_{n}: X \rightarrow X$ via the mappings $h, f_{n}, r$ and the locally Lipschitz pseudo-gradient vector field $v$ as

$$
g_{n}(u)= \begin{cases}-f_{n}(u) h(u) r\left(\|v(u)\|_{X}\right) v(u), & u \in X \backslash \Gamma, \\ 0, & u \in \Gamma .\end{cases}
$$

Thus, the mapping $g_{n}$ is locally Lipschitz on $X$ (thanks to the definition of $h$ and $f_{n}$ ) and $\left\|g_{n}(u)\right\|_{X} \leq 1$ for all $u \in X$.

We consider the following initial value problem on the Banach space $X$ with an initial condition $u \in X$ which defines a minus-gradient flow for the functional $F$

$$
\left\{\begin{array}{l}
\frac{\mathrm{d} w(t)}{\mathrm{d} t}=g_{n}(w(t)), \quad t \in[0, \infty) \\
w(0)=u
\end{array}\right.
$$

The construction of the minus-gradient flow (4.9) is adopted from P. H. Rabinowitz [31, Thm. A.4] where one can find also the proof of the following statement.

Lemma 4.2. Let the assumptions of Thm. 1.1 and (4.1) be satisfied and $n \in \mathbb{N}$ be given. Then the following hold:

(i) For every given $u \in X$ there exists a unique solution of (4.9) and it exists for all $t \in[0, \infty)$.

(ii) Denoting by $w_{n}(t, u)$ the image of the unique solution of (4.9) with given $u \in X$ at $t \in[0, \infty)$, the operator $w_{n}:[0, \infty) \times X \rightarrow X$ is continuous on $[0, \infty) \times X$.

(iii) The function $F\left(w_{n}(\cdot, u)\right):[0, \infty) \rightarrow \mathbb{R}$ is decreasing for every given $u \in X$. 
In the rest of this section we analyze the restriction of the mapping $w_{n}$ from Lem. 4.2 (ii) to $[0, \infty) \times \partial B_{Y}(R)$. In other words we deform the finite dimensional sphere $\partial B_{Y}(R) \subset Y$ into the whole space $X$ by the minus-gradient flow (4.9). We find a third critical point of $F$ via this deformation which contradicts (4.1).

Since $\partial B_{Y}(R)$ is a compact set and $F\left(w_{n}(t, \cdot)\right): \partial B_{Y}(R) \rightarrow \mathbb{R}$ is continuous for every given $t \in[0, \infty)$, there exists

$$
\max _{u \in \partial B_{Y}(R)} F\left(w_{n}(t, u)\right) \text { for every } t \in[0, \infty) .
$$

Lemma 4.3. Let the assumptions of Thm. 1.1 and (4.1) be satisfied and $n \in \mathbb{N}$ be given. Then the limit

$$
c_{n}=\lim _{t \rightarrow \infty} \max _{u \in \partial B_{Y}(R)} F\left(w_{n}(t, u)\right)
$$

exists, can be also characterized as $c_{n}=\inf _{t \in[0, \infty)} \max _{u \in \partial B_{Y}(R)} F\left(w_{n}(t, u)\right)$ and satisfies $c_{n} \leq a$.

Proof. Consider the function $\varphi:[0, \infty) \rightarrow \mathbb{R}$ defined as

$$
\varphi(t)=\max _{u \in \partial B_{Y}(R)} F\left(w_{n}(t, u)\right) .
$$

The function $\varphi$ is bounded from below, since the functional $F$ is bounded from below as well. Moreover, $\varphi$ is decreasing. Indeed, let $t_{1}<t_{2}$. Using the compactness of the set $\partial B_{Y}(R)$ and the continuity of the mapping $F\left(w_{n}(t, \cdot)\right)$ for every given $t \in[0, \infty)$, we find $u_{1}, u_{2} \in \partial B_{Y}(R)$ such that

$$
F\left(w_{n}\left(t_{1}, u_{1}\right)\right)=\max _{u \in \partial B_{Y}(R)} F\left(w_{n}\left(t_{1}, u\right)\right), \quad F\left(w_{n}\left(t_{2}, u_{2}\right)\right)=\max _{u \in \partial B_{Y}(R)} F\left(w_{n}\left(t_{2}, u\right)\right) .
$$

Then using the definition of $u_{1}$ and the fact that $F\left(w_{n}\left(\cdot, u_{2}\right)\right)$ is decreasing by Lem. 4.2 (iii), we obtain the following estimate

$$
\varphi\left(t_{1}\right)=F\left(w_{n}\left(t_{1}, u_{1}\right)\right) \geq F\left(w_{n}\left(t_{1}, u_{2}\right)\right) \geq F\left(w_{n}\left(t_{2}, u_{2}\right)\right)=\varphi\left(t_{2}\right) .
$$

Thus, $\varphi$ is bounded from below and decreasing. This implies that there exists $c_{n} \in \mathbb{R}$ such that

$$
c_{n}=\lim _{t \rightarrow \infty} \varphi(t)=\inf _{t \in[0, \infty)} \varphi(t) \leq \varphi(0)=a .
$$

We distinct two qualitatively different cases in the proof of the existence of a third critical point - the existence of a second global minimizer or a second minimax. This corresponds to the following two possibilities, respectively:

(i) for all $n \in \mathbb{N}$ there is $c_{n} \leq F\left(u_{M}\right)+\frac{1}{n}$,

(ii) there exists $n_{0} \in \mathbb{N}$ such that $c_{n_{0}}>F\left(u_{M}\right)+\frac{1}{n_{0}}$,

where $c_{n} \in \mathbb{R}$ are defined by Lem. 4.3. We treat these two cases separately for the sake of lucidity.

\subsection{Second global minimizer}

In this subsection we analyze the case in which

$$
c_{n} \leq F\left(u_{M}\right)+\frac{1}{n} \quad \text { for all } n \in \mathbb{N}
$$

From the decomposition of the space $X$ into $X=Y \oplus Z$, there exist unique $y_{M} \in Y$ and $z_{M} \in Z$ such that the global minimizer $u_{M} \in X$ of $F$ given by Lem. 4.1 satisfies

$$
u_{M}=y_{M}+z_{M} .
$$

Since $F\left(u_{M}\right)<b$, there is $u_{M} \notin Z$ or equivalently $y_{M} \neq o$. Hence, there exists $\delta>0$ sufficiently small such that

$$
o \notin \bar{B}_{Y}\left(y_{M}, \delta\right),
$$

where $\bar{B}_{Y}\left(y_{M}, \delta\right)$ denotes the closed ball in the subspace $Y$ with the radius $\delta>0$ centered at $y_{M} \in Y$.

Moreover, let $P: X \rightarrow Y$ be the projection of $X$ onto $Y$ along $Z$. Thus, $P$ is a linear mapping such that ker $P=Z$ and thanks to the finite dimension of $Y$ also continuous (see, e.g., P. Drábek, J. Milota [12, Rem. 2.1.19]). Obviously, $P\left(u_{M}\right)=y_{M}$.

Lemma 4.4. Let the assumptions of Thm. 1.1 and (4.1) be satisfied, $c_{n} \leq F\left(u_{M}\right)+\frac{1}{n}$ for all $n \in \mathbb{N}$ and $\delta>0$ be sufficiently small that $o \notin \bar{B}_{Y}\left(y_{M}, \delta\right)$. Then for every $n \in \mathbb{N}$ there exist $t_{n} \in[0, \infty)$ and $u_{n} \in \partial B_{Y}(R)$ such that

$$
F\left(w_{n}\left(t_{n}, u_{n}\right)\right) \leq F\left(u_{M}\right)+\frac{2}{n} \quad \text { and } \quad\left\|P\left(w_{n}\left(t_{n}, u_{n}\right)\right)-y_{M}\right\|_{X}>\delta .
$$


Proof. Assume by contradiction that there exists $\tilde{n} \in \mathbb{N}$ such that for all $t \in[0, \infty)$ and $u \in \partial B_{Y}(R)$ there is

$$
F\left(w_{\tilde{n}}(t, u)\right)>F\left(u_{M}\right)+\frac{2}{\tilde{n}} \quad \text { or } \quad\left\|P\left(w_{\tilde{n}}(t, u)\right)-y_{M}\right\|_{X} \leq \delta .
$$

From the definition of $c_{\tilde{n}}$ and from the assumption that $c_{\tilde{n}} \leq F\left(u_{M}\right)+\frac{1}{\tilde{n}}$ there has to exist $\tilde{t} \in[0, \infty)$ such that for all $u \in \partial B_{Y}(R)$ there is

$$
F\left(w_{\tilde{n}}(\tilde{t}, u)\right) \leq \max _{u \in \partial B_{Y}(R)} F(\tilde{t}, u) \leq F\left(u_{M}\right)+\frac{2}{\tilde{n}} .
$$

This implies that for $t=\tilde{t}$ the former inequality in (4.10) does not hold for any $u \in \partial B_{Y}(R)$ and thus, the latter one has to be true,

$$
\left\|P\left(w_{\tilde{n}}(\tilde{t}, u)\right)-y_{M}\right\|_{X} \leq \delta \quad \text { for all } u \in \partial B_{Y}(R) .
$$

Applying Lem. 3.1 with $K=\bar{B}_{Y}\left(y_{M}, \delta\right)$ for the continuous mapping $P \circ w_{\tilde{n}}:[0, \tilde{t}] \times \partial B_{Y}(R) \rightarrow Y$, we obtain that there exist $t_{0} \in(0, \tilde{t})$ and $u_{0} \in \partial B_{Y}(R)$ such that $P\left(w_{\tilde{n}}\left(t_{0}, u_{0}\right)\right)=o$. Then

$$
w_{\tilde{n}}\left(t_{0}, u_{0}\right) \in Z
$$

because of $\operatorname{ker} P=Z$. This is a contradiction, since the following inequality holds using the fact that the function $F\left(w_{\tilde{n}}\left(\cdot, u_{0}\right)\right)$ is decreasing by Lem. 4.2 (iii)

$$
F\left(w_{\tilde{n}}\left(t_{0}, u_{0}\right)\right) \leq F\left(w_{\tilde{n}}\left(0, u_{0}\right)\right)=F\left(u_{0}\right) \leq a<b=\inf _{u \in Z} F(u) .
$$

Lem. 4.4 guarantees the existence of a sequence $\left\{w_{n}\left(t_{n}, u_{n}\right)\right\} \subset X$ such that

$$
F\left(w_{n}\left(t_{n}, u_{n}\right)\right) \leq F\left(u_{M}\right)+\frac{2}{n} \text { and }\left\|P\left(w_{n}\left(t_{n}, u_{n}\right)\right)-y_{M}\right\|_{X}>\delta \text { for all } n \in \mathbb{N} .
$$

Particularly, there is $F\left(w_{n}\left(t_{n}, u_{n}\right)\right) \rightarrow F\left(u_{M}\right)=\min _{u \in X} F(u)$ for $n \rightarrow \infty$. Moreover, the following inequalities hold for all $n \in \mathbb{N}$

$$
\delta<\left\|P\left(w_{n}\left(t_{n}, u_{n}\right)\right)-y_{M}\right\|_{X}=\left\|P\left(w_{n}\left(t_{n}, u_{n}\right)-u_{M}\right)\right\|_{X} \leq\|P\|_{\mathcal{L}(X, Y)}\left\|w_{n}\left(t_{n}, u_{n}\right)-u_{M}\right\|_{X} .
$$

Since $Y \neq\{o\}$, the projection $P$ given by the decomposition $X=Y \oplus Z$ satisfies $\|P\|_{\mathcal{L}(X, Y)} \geq 1$ and there is

$$
\left\|w_{n}\left(t_{n}, u_{n}\right)-u_{M}\right\|_{X}>\frac{\delta}{\|P\|_{\mathcal{L}(X, Y)}}>0 \quad \text { for all } n \in \mathbb{N} .
$$

Consequently, $\left\{w_{n}\left(t_{n}, u_{n}\right)\right\} \subset X$ is a minimizing sequence and Thm. 2.4 yields that there exists a sequence $\left\{\nu_{n}\right\} \subset X$ such that:

(i) $F\left(\nu_{n}\right) \leq F\left(w_{n}\left(t_{n}, u_{n}\right)\right)$ for all $n \in \mathbb{N}$,

(ii) $\left\|w_{n}\left(t_{n}, u_{n}\right)-\nu_{n}\right\|_{X} \rightarrow 0$,

(iii) $\left\|F^{\prime}\left(\nu_{n}\right)\right\|_{X^{*}} \rightarrow 0$.

Taking into account the statements (i) and (iii) and the Palais-Smale condition (PS), we obtain

$$
\nu_{n} \rightarrow \nu_{0} \in X \quad \text { (at least for a subsequence). }
$$

Since $F \in C^{1}(X, \mathbb{R})$, the statements (i) and (iii) yield moreover

$$
F\left(\nu_{n}\right) \rightarrow F\left(\nu_{0}\right)=F\left(u_{M}\right)=\min _{u \in X} F(u) \quad \text { and } \quad F^{\prime}\left(\nu_{n}\right) \rightarrow F^{\prime}\left(\nu_{0}\right)=o .
$$

Hence, $\nu_{0}$ is a global minimizer and thus, a critical point of $F$. Furthermore, the statement (ii) yields

$$
\left\|w_{n}\left(t_{n}, u_{n}\right)-\nu_{0}\right\|_{X} \leq\left\|w_{n}\left(t_{n}, u_{n}\right)-\nu_{n}\right\|_{X}+\left\|\nu_{n}-\nu_{0}\right\|_{X} \rightarrow 0 .
$$

Since $\left\|w_{n}\left(t_{n}, u_{n}\right)-u_{M}\right\|_{X}>\frac{\delta}{\|P\|_{\mathcal{L}(X, Y)}}>0$ for all $n \in \mathbb{N}$ by $(4.13)$, there is

and consequently, $\nu_{0} \neq u_{M}$.

$$
\left\|\nu_{0}-u_{M}\right\|_{X} \geq \frac{\delta}{\|P\|_{\mathcal{L}(X, Y)}}>0
$$

This is a contradiction with the assumption (4.1) that $u_{M}$ and $u_{S}$ are the only critical points of $F$. 


\subsection{Second minimax}

In this subsection we analyze the latter case in which there exists $n_{0} \in \mathbb{N}$ such that

$$
c_{n_{0}}>F\left(u_{M}\right)+\frac{1}{n_{0}} .
$$

Let us denote for the simplicity $c_{0}=c_{n_{0}}$ and $w_{0}=w_{n_{0}}$, i.e., we suppose

$$
c_{0}=\lim _{t \rightarrow \infty} \max _{u \in \partial B_{Y}(R)} F\left(w_{0}(t, u)\right)>F\left(u_{M}\right)+\frac{1}{n_{0}} .
$$

Firstly, we show the following lemma about the realization of $c_{0}$ at a point $u_{0} \in \partial B_{Y}(R)$.

Lemma 4.5. Let the assumptions of Thm. 1.1 and (4.1) be satisfied and $n_{0} \in \mathbb{N}$ be given. Then there exists $u_{0} \in \partial B_{Y}(R)$ such that

$$
c_{0}=\lim _{t \rightarrow \infty} F\left(w_{0}\left(t, u_{0}\right)\right) .
$$

Proof. The following inequality holds using the characterization of $c_{0}$ via the infimum from Lem. 4.3 and standard properties of infimum and supremum

$$
\begin{aligned}
c_{0} & =\lim _{t \rightarrow \infty} \max _{u \in \partial B_{Y}(R)} F\left(w_{0}(t, u)\right)=\inf _{t \in[0, \infty)} \max _{u \in \partial B_{Y}(R)} F\left(w_{0}(t, u)\right) \\
& \geq \sup _{u \in \partial B_{Y}(R)} \inf _{t \in[0, \infty)} F\left(w_{0}(t, u)\right)=\sup _{u \in \partial B_{Y}(R)} \lim _{t \rightarrow \infty} F\left(w_{0}(t, u)\right) .
\end{aligned}
$$

We assume by contradiction that the statement of lemma does not hold, i.e.,

$$
\lim _{t \rightarrow \infty} F\left(w_{0}(t, u)\right)<c_{0} \quad \text { for all } \quad u \in \partial B_{Y}(R) .
$$

Firstly, we show that under the assumption (4.14) there is

$$
\sup _{u \in \partial B_{Y}(R)} \lim _{t \rightarrow \infty} F\left(w_{0}(t, u)\right)<c_{0} .
$$

Suppose that the contrary holds true, i.e., that (4.14) is satisfied and

$$
\sup _{u \in \partial B_{Y}(R)} \lim _{t \rightarrow \infty} F\left(w_{0}(t, u)\right)=c_{0} .
$$

Thus, there exists a sequence $\left\{u_{k}\right\} \subset \partial B_{Y}(R)$ such that

$$
\lim _{k \rightarrow \infty} \lim _{t \rightarrow \infty} F\left(w_{0}\left(t, u_{k}\right)\right)=c_{0} .
$$

Since $\partial B_{Y}(R)$ is a compact set, there is $u_{k} \rightarrow \tilde{u}$ for some $\tilde{u} \in \partial B_{Y}(R)$ (at least for a subsequence). From (4.14) we obtain $\lim _{t \rightarrow \infty} F\left(w_{0}(t, \tilde{u})\right)=c_{0}-\varepsilon<c_{0}$ for an $\varepsilon>0$. Hence, there exists $\tilde{t} \in[0, \infty)$ such that $F\left(w_{0}(\tilde{t}, \tilde{u})\right)<c_{0}-\frac{\varepsilon}{2}$. Since the mapping $F\left(w_{0}(\tilde{t}, \cdot)\right)$ is continuous, there exists a ball $B(\tilde{u}, \delta)$ with a sufficiently small radius $\delta>0$ such that

$$
F\left(w_{0}(\tilde{t}, u)\right)<c_{0}-\frac{\varepsilon}{2} \quad \text { for all } \quad u \in B(\tilde{u}, \delta) \cap \partial B_{Y}(R)
$$

The convergence $u_{k} \rightarrow \tilde{u}$ provides the existence of $\tilde{k} \in \mathbb{N}$ such that $u_{k} \in B(\tilde{u}, \delta) \cap \partial B_{Y}(R)$ for all $k \geq \tilde{k}$. Since the functions $F\left(w_{0}\left(\cdot, u_{k}\right)\right)$ are decreasing by Lem. 4.2 (iii), there is

$$
F\left(w_{0}\left(t, u_{k}\right)\right) \leq F\left(w_{0}\left(\tilde{t}, u_{k}\right)\right)<c_{0}-\frac{\varepsilon}{2} \quad \text { for all } t \geq \tilde{t} \text { and } k \geq \tilde{k} .
$$

At this place we pass to a limit for $t \rightarrow \infty$ and then to a limit superior for $k \rightarrow \infty$ in (4.17) to obtain

$$
\limsup _{k \rightarrow \infty} \lim _{t \rightarrow \infty} F\left(w_{0}\left(t, u_{k}\right)\right) \leq c_{0}-\frac{\varepsilon}{2}<c_{0}
$$

a contradiction with (4.16). Consequently, under the assumption (4.14), the inequality (4.15) holds.

Denote

$$
S=\sup _{u \in \partial B_{Y}(R)} \lim _{t \rightarrow \infty} F\left(w_{0}(t, u)\right)
$$

Since $F\left(w_{0}(\cdot, u)\right)$ are continuous, decreasing and $S<c_{0}$ by $(4.15)$, then for all $u \in \partial B_{Y}(R)$ there exists

$$
\xi(u)=\inf \left\{t \in[0, \infty): F\left(w_{0}(\tau, u)\right)<\frac{S+c_{0}}{2} \text { for all } \tau>t\right\} .
$$

The mapping $\xi: \partial B_{Y}(R) \rightarrow[0, \infty)$ is upper semicontinuous on the compact set $\partial B_{Y}(R)$, i.e., 


$$
\limsup _{k \rightarrow \infty} \xi\left(u_{k}\right) \leq \xi(\tilde{u})
$$

for all sequences $\left\{u_{k}\right\} \in \partial B_{Y}(R)$ such that $u_{k} \rightarrow \tilde{u}$ for some $\tilde{u} \in \partial B_{Y}(R)$. Indeed, let $\left\{u_{k}\right\} \subset \partial B_{Y}(R)$ and $u_{k} \rightarrow \tilde{u}$. We split the sequence $\left\{\xi\left(u_{k}\right)\right\} \subset[0, \infty)$ into two parts

$$
\begin{gathered}
\left\{\xi\left(u_{k_{m}}\right)\right\} \text { such that } \xi\left(u_{k_{m}}\right)>\xi(\tilde{u}) \text { for all } m \in \mathbb{N}, \\
\left\{\xi\left(u_{k_{l}}\right)\right\} \text { such that } \xi\left(u_{k_{l}}\right) \leq \xi(\tilde{u}) \text { for all } l \in \mathbb{N} .
\end{gathered}
$$

Note that if one of these parts is a finite set, we can treat only with the other part, i.e., we suppose without loss of generality that both parts are infinite subsequences of $\left\{\xi\left(u_{k}\right)\right\}$. There is obviously

$$
\limsup _{l \rightarrow \infty} \xi\left(u_{k_{l}}\right) \leq \xi(\tilde{u}) .
$$

Let us show that also $\limsup _{m \rightarrow \infty} \xi\left(u_{k_{m}}\right) \leq \xi(\tilde{u})$. Assume the contrary, i.e., there exists $\varepsilon>0$ such that

$$
\xi\left(u_{k_{m}}\right) \geq \xi(\tilde{u})+\varepsilon \quad \text { (at least for a subsequence). }
$$

Since $F\left(w_{0}(\cdot, \tilde{u})\right)$ is decreasing, the definition of $\xi(\tilde{u})$ implies

$$
F\left(w_{0}(\xi(\tilde{u})+\varepsilon, \tilde{u})\right)<\frac{S+c_{0}}{2} .
$$

The continuity of $F\left(w_{0}(\xi(\tilde{u})+\varepsilon, \cdot)\right)$ and the convergence $u_{k_{m}} \rightarrow \tilde{u}$ yield that for sufficiently large $m \in \mathbb{N}$ there is

$$
F\left(w_{0}\left(\xi(\tilde{u})+\varepsilon, u_{k_{m}}\right)\right)<\frac{S+c_{0}}{2},
$$

a contradiction with the definition of $\xi\left(u_{k_{m}}\right),(4.18)$ and the continuity of $F\left(w_{0}\left(\cdot, u_{k_{m}}\right)\right)$. Therefore, the mapping $\xi: \partial B_{Y}(R) \rightarrow[0, \infty)$ is upper semicontinuous on the compact set $\partial B_{Y}(R)$. This yields that there exists

$$
\bar{\xi}=\max _{u \in \partial B_{Y}(R)} \xi(u)<\infty
$$

(see, e.g., P. Drábek, J. Milota [12, p. 451]). The functions $F\left(w_{0}(\cdot, u)\right)$ are decreasing by Lem. 4.2 (iii) and thus,

This implies that

$$
F\left(w_{0}(t, u)\right) \leq \frac{S+c_{0}}{2} \text { for all } t \geq \bar{\xi} \text { and } u \in \partial B_{Y}(R)
$$

$$
\lim _{t \rightarrow \infty} \max _{u \in \partial B_{Y}(R)} F\left(w_{0}(t, u)\right) \leq \frac{S+c_{0}}{2}<c_{0},
$$

a final contradiction with the definition of $c_{0}$. Therefore, (4.14) is not valid and the statement of lemma holds.

Consequently, if there exists $n_{0} \in \mathbb{N}$ such that $c_{0}>F\left(u_{M}\right)+\frac{1}{n_{0}}$, Lem. 4.5 guarantees the existence of $u_{0} \in$ $\partial B_{Y}(R)$ such that

$$
\lim _{t \rightarrow \infty} F\left(w_{0}\left(t, u_{0}\right)\right)=c_{0}>F\left(u_{M}\right)+\frac{1}{n_{0}} .
$$

Since $F\left(w_{0}\left(\cdot, u_{0}\right)\right)$ is decreasing by Lem. 4.2 (iii), the following hold

$$
\begin{gathered}
F\left(w_{0}\left(t, u_{0}\right)\right) \leq F\left(w_{0}\left(0, u_{0}\right)\right)=F\left(u_{0}\right) \leq a<\frac{a+b}{2}, \quad \text { i.e., } \quad w_{0}\left(t, u_{0}\right) \notin H \text { for all } t \in[0, \infty), \\
F\left(w_{0}\left(t, u_{0}\right)\right) \geq c_{0}>F\left(u_{M}\right)+\frac{1}{n_{0}}, \quad \text { i.e., } \quad w_{0}\left(t, u_{0}\right) \notin B_{n_{0}} \quad \text { for all } t \in[0, \infty),
\end{gathered}
$$

where the sets $H$ and $B_{n_{0}}$ are defined in (4.4) and (4.5), respectively. This yields that $h\left(w_{0}\left(t, u_{0}\right)\right)=1$ and $f_{n_{0}}\left(w_{0}\left(t, u_{0}\right)\right)=1$ for all $t \in[0, \infty)$ (the mappings $h$ and $f_{n_{0}}$ are defined in (4.6)). Thus, $w_{0}\left(\cdot, u_{0}\right)$ is the unique solution of the following initial value problem

$$
\left\{\begin{array}{l}
\frac{\mathrm{d} w_{0}\left(t, u_{0}\right)}{\mathrm{d} t}=-r\left(\left\|v\left(w_{0}\left(t, u_{0}\right)\right)\right\|_{X}\right) v\left(w_{0}\left(t, u_{0}\right)\right), \quad t \in[0, \infty), \\
w_{0}\left(0, u_{0}\right)=u_{0} .
\end{array}\right.
$$

Lemma 4.6. Let the assumptions of Thm. 1.1 and (4.1) be satisfied, there exist $n_{0} \in \mathbb{N}$ such that $c_{0}>F\left(u_{M}\right)+\frac{1}{n_{0}}$ and $u_{0} \in \partial B_{Y}(R)$ be as in Lem. 4.5. Then there exists a sequence $\left\{t_{k}\right\} \in[0, \infty)$ such that:

(i) $c_{0} \leq F\left(w_{0}\left(t_{k}, u_{0}\right)\right) \leq$ a for all $k \in \mathbb{N}$,

(ii) $\left\|F^{\prime}\left(w_{0}\left(t_{k}, u_{0}\right)\right)\right\|_{X^{*}} \rightarrow 0$. 
Proof. The statement (i) holds for all sequences $\left\{t_{k}\right\} \in[0, \infty)$, since the unique solution $w_{0}\left(\cdot, u_{0}\right)$ of $(4.19)$ satisfies $c_{0} \leq F\left(w_{0}\left(t, u_{0}\right)\right) \leq a$ for all $t \in[0, \infty)$. Assume by contradiction that (ii) does not hold, i.e., there exists $\varepsilon>0$ such that for all $t \in[0, \infty)$ there is

$$
\left\|F^{\prime}\left(w_{0}\left(t, u_{0}\right)\right)\right\|_{X^{*}} \geq \varepsilon
$$

The following estimate holds using the chain rule (see, e.g., P. Drábek, J. Milota [12, Cor. 3.2.13]), (4.19) and the inequality (4.3) for the pseudo-gradient vector field $v$

$$
\begin{aligned}
F\left(w_{0}\left(t, u_{0}\right)\right) & =F\left(w_{0}\left(0, u_{0}\right)\right)+\int_{0}^{t} \frac{\mathrm{d} F\left(w_{0}\left(\tau, u_{0}\right)\right)}{\mathrm{d} \tau} \mathrm{d} \tau \\
& =F\left(u_{0}\right)-\int_{0}^{t} r\left(\left\|v\left(w_{0}\left(\tau, u_{0}\right)\right)\right\|_{X}\right)\left\langle F^{\prime}\left(w_{0}\left(\tau, u_{0}\right)\right), v\left(w_{0}\left(\tau, u_{0}\right)\right)\right\rangle \mathrm{d} \tau \\
& \leq F\left(u_{0}\right)-\int_{0}^{t} r\left(\left\|v\left(w_{0}\left(\tau, u_{0}\right)\right)\right\|_{X}\right)\left\|F^{\prime}\left(w_{0}\left(\tau, u_{0}\right)\right)\right\|_{X^{*}}^{2} \mathrm{~d} \tau .
\end{aligned}
$$

Let us distinguish between two cases for an arbitrary $\tau \in[0, \infty)$. Firstly, if $\left\|v\left(w_{0}\left(\tau, u_{0}\right)\right)\right\|_{X} \leq 1$, there is $r\left(\left\|v\left(w_{0}\left(\tau, u_{0}\right)\right)\right\|_{X}\right)=1$ by the definition of function $r$ (4.7). Hence, (4.20) implies that

$$
r\left(\left\|v\left(w_{0}\left(\tau, u_{0}\right)\right)\right\|_{X}\right)\left\|F^{\prime}\left(w_{0}\left(\tau, u_{0}\right)\right)\right\|_{X^{*}}^{2}=\left\|F^{\prime}\left(w_{0}\left(\tau, u_{0}\right)\right)\right\|_{X^{*}}^{2} \geq \varepsilon^{2} .
$$

Secondly, if $\left\|v\left(w_{0}\left(\tau, u_{0}\right)\right)\right\|_{X}>1$, then by $(4.7)$

$$
r\left(\left\|v\left(w_{0}\left(\tau, u_{0}\right)\right)\right\|_{X}\right)=\frac{1}{\left\|v\left(w_{0}\left(\tau, u_{0}\right)\right)\right\|_{X}} .
$$

The inequality (4.2) for the pseudo-gradient vector field $v$ and (4.20) yield the following estimate

$$
r\left(\left\|v\left(w_{0}\left(\tau, u_{0}\right)\right)\right\|_{X}\right)\left\|F^{\prime}\left(w_{0}\left(\tau, u_{0}\right)\right)\right\|_{X^{*}}^{2}=\frac{\left\|F^{\prime}\left(w_{0}\left(\tau, u_{0}\right)\right)\right\|_{X^{*}}^{2}}{\left\|v\left(w_{0}\left(\tau, u_{0}\right)\right)\right\|_{X}} \geq \frac{\left\|F^{\prime}\left(w_{0}\left(\tau, u_{0}\right)\right)\right\|_{X^{*}}^{2}}{2\left\|F^{\prime}\left(w_{0}\left(\tau, u_{0}\right)\right)\right\|_{X^{*}}} \geq \frac{\varepsilon}{2} .
$$

Putting (4.22) and (4.23) together, we obtain that

$$
r\left(\left\|v\left(w_{0}\left(\tau, u_{0}\right)\right)\right\|_{X}\right)\left\|F^{\prime}\left(w_{0}\left(\tau, u_{0}\right)\right)\right\|_{X^{*}}^{2} \geq \alpha=\min \left\{\varepsilon^{2}, \frac{\varepsilon}{2}\right\}>0 \quad \text { for all } \quad \tau \in[0, \infty) .
$$

Consequently, we can continue in the estimate (4.21) as follows

This yields that

$$
\begin{aligned}
F\left(w_{0}\left(t, u_{0}\right)\right) & \leq F\left(u_{0}\right)-\int_{0}^{t} r\left(\left\|v\left(w_{0}\left(\tau, u_{0}\right)\right)\right\|_{X}\right)\left\|F^{\prime}\left(w_{0}\left(\tau, u_{0}\right)\right)\right\|_{X^{*}}^{2} \mathrm{~d} \tau \\
& \leq F\left(u_{0}\right)-\int_{0}^{t} \alpha \mathrm{d} \tau \\
& =F\left(u_{0}\right)-\alpha t
\end{aligned}
$$

$$
\lim _{t \rightarrow \infty} F\left(w_{0}\left(t, u_{0}\right)\right)=-\infty
$$

which is a contradiction with the statement of Lem. 4.5 that $\lim _{t \rightarrow \infty} F\left(w_{0}\left(t, u_{0}\right)\right)=c_{0}$ (apparently, this also contradicts the assumption that $F$ is bounded from below).

Lem. 4.6 guarantees the existence of a Palais-Smale sequence $\left\{w_{0}\left(t_{k}, u_{0}\right)\right\} \subset X$. Hence, applying the PalaisSmale condition $(P S)$ we obtain that

Since $F \in C^{1}(X, \mathbb{R})$, there is

$$
w_{0}\left(t_{k}, u_{0}\right) \rightarrow \nu_{0} \in X \quad \text { (at least for a subsequence) }
$$

$$
F\left(w_{0}\left(t_{k}, u_{0}\right)\right) \rightarrow F\left(\nu_{0}\right) \in\left[c_{0}, a\right] \quad \text { and } \quad F^{\prime}\left(w_{0}\left(t_{k}, u_{0}\right)\right) \rightarrow F^{\prime}\left(\nu_{0}\right)=o .
$$

Therefore, $\nu_{0}$ is a critical point of $F$. Moreover, $\nu_{0} \neq u_{M}$ and $\nu_{0} \neq u_{S}$, because of $F\left(u_{M}\right)<c_{0} \leq a<b \leq F\left(u_{S}\right)$.

This yields a final contradiction with the assumption (4.1). The proof of Thm. 1.1 is complete.

\section{Application for semilinear stationary PDE}

In this section we present an application of Thm. 1.1 for semilinear stationary PDEs. We study the following Dirichlet boundary value problem involving superlinear power-function and small spatial perturbation

$$
\begin{cases}-\Delta u(x)=\lambda u(x)-|u(x)|^{p-2} u(x)+f(x), & x \in \Omega, \\ u(x)=0, & x \in \partial \Omega,\end{cases}
$$

where $\Omega \subset \mathbb{R}^{N}$ is an open, bounded domain with a smooth boundary, $\Delta u=\operatorname{div}(\nabla u)$ is the $N$-dimensional Laplacian, $\lambda>0$ and $p$ satisfies: 
(i) $2<p$ if $N=1,2$,

(ii) $2<p<\frac{2 N}{N-2}$ if $N \geq 3$.

We study the existence of at least three weak solutions of (5.1). The problem (5.1) is widely studied in the theory of nonlinear PDEs. It describes stationary (or steady state) solutions of the following reaction-diffusion equation

$$
\begin{cases}\frac{\partial u(x, t)}{\partial t}-\Delta_{x} u(x, t)=\lambda u(x, t)-|u(x, t)|^{p-2} u(x, t)+f(x), & x \in \Omega, \quad t \in\left[t_{0}, \infty\right), \\ u\left(x, t_{0}\right)=u_{0}(x), & x \in \Omega, \\ u(x, t)=0, & x \in \partial \Omega, \quad t \in\left[t_{0}, \infty\right) .\end{cases}
$$

The initial-boundary value problem (5.2) is related to the so-called bistable equation which is used in physics as a model of phase transitions. As an example we can mention the case of $p=4$ which was studied in 1979 by S. M. Allen and J. W. Cahn in [1] for modeling of phase separation in binary alloy systems. This problem is therefore often called the Allen-Cahn equation. For the study of stationary problem for the bistable equation with Dirichlet boundary condition see, e.g., M. S. Berger, L. E. Fraenkel [3] or N. C. Owen, J. Rubinstein, P. Sternberg [27]. Let us mention that there are also many works dealing with (5.1) or (5.2) with Neumann boundary condition, see, e.g., references in N. C. Owen, J. Rubinstein, P. Sternberg [27].

From another point of view, the reaction-diffusion equation (5.2) is motivated from the population biology. For example for $p=3$ its positive solutions describe the evolution of population density of single species whose reproduction follows the logistic law and which homogeneously diffuses in the environment with hostile boundary, see J. D. Murray [25, Sec. 11.2]. This problem is often called the Fischer or Fischer-Kolmogorov-Petrovskii-Piskunov equation after R. A. Fischer, who proposed the problem in 1937 for the modeling of spatial spread of a gene in the population [14], and A. Kolmogorov, I. Petrovskii and N. Piskunov, who presented the first fundamental results [18].

The original Fischer equation assumes no spatial perturbation, i.e., $f(x) \equiv 0$ in $(5.2)$. This problem is widely studied, see, e.g., D. Henry [16]. However, from the biological point of view (e.g., fishery management problems) there are natural reasons why the spatial sources or sinks should be involved, for example predation or harvesting of the species could happen. The problems with logistic growth and constant yield harvesting modeled by $f(x)<0$, $x \in \Omega$, in (5.2) or its stationary version (5.1) have been recently studied in many papers, see, e.g., S. Oruganti, J. Shi, R. Shivaji [26] or P. Liu, J. Shi, Y. Wang [21].

The problem (5.1) with $f(x)<0, x \in \Omega$, is an example of the so-called semipositone problem. Semipositone problems were introduced by A. Castro and R. Shivaji in [8] as boundary value problems for the following PDE

$$
-\Delta u(x)=g(x, u), \quad x \in \Omega,
$$

where the right-hand side is not positone, specifically, satisfies $g(x, 0)<0, x \in \Omega$. For an overview about semipositone problems see A. Castro, C. Maya, R. Shivaji [9]. They point out that these problems could be mathematically challenging. For example, the existence of positive solutions which is natural specifically in biological models is intensively studied (see, e.g., S. Oruganti, J. Shi, R. Shivaji [26], P. Liu, J. Shi, Y. Wang [21] again).

We denote by $\lambda_{k}, k \in \mathbb{N}$, the $k$-th eigenvalue of the operator $-\Delta$ on $\Omega$ with the homogeneous Dirichlet boundary condition (involving multiplicities). We consider a nonresonant case of (5.1) in which the parameter $\lambda>0$ satisfies $\lambda_{k}<\lambda<\lambda_{k+1}$ for some $k \in \mathbb{N}$. Assuming $f \in L^{2}(\Omega)$ we apply Thm. 1.1 to show the existence of multiple weak solutions of (5.1). The following definition is well-known (see, e.g., P. Drábek, J. Milota [12, Sec. 5.9A]).

Definition 5.1. The weak solution of $(5.1)$ is a function $u \in W_{0}^{1,2}(\Omega)$ such that the following equality holds for any $v \in W_{0}^{1,2}(\Omega)$

$$
\int_{\Omega} \nabla u(x) \cdot \nabla v(x) \mathrm{d} x=\lambda \int_{\Omega} u(x) v(x) \mathrm{d} x-\frac{1}{p} \int_{\Omega}|u(x)|^{p-2} u(x) v(x) \mathrm{d} x+\int_{\Omega} f(x) v(x) \mathrm{d} x .
$$

Remark 5.2. One can note that if we assume instead of $f \in L^{2}(\Omega)$ a stronger assumption, e.g., that $f$ is Lipschitz on $\bar{\Omega}$, then every weak solution of (5.1) is also a classical solution (see, e.g., P. H. Rabinowitz [31, Rem. 2.22]).

We use the equivalent norm on the Sobolev space $W_{0}^{1,2}(\Omega)$

$$
\|u\|=\left(\int_{\Omega}|\nabla u(x)|^{2} \mathrm{~d} x\right)^{\frac{1}{2}}, \quad u \in W_{0}^{1,2}(\Omega),
$$

and the $p$-norm on the Lebesgue spaces $L^{p}(\Omega), p \geq 1$,

$$
\|u\|_{p}=\left(\int_{\Omega}|u(x)|^{p} \mathrm{~d} x\right)^{\frac{1}{p}}, \quad u \in L^{p}(\Omega) .
$$

In the rest of the section we prove the following theorem about the existence of at least three weak solutions of (5.1) in the nonresonant case. 
Theorem 5.3. Let $\lambda_{k}<\lambda<\lambda_{k+1}$. Then there exists $\mu>0$ such that the boundary value problem (5.1) possesses at least three weak solutions for all $f \in L^{2}(\Omega)$ with $\|f\|_{2}<\mu$.

The problem (5.1) with logistic nonlinearity and constant yield harvesting $f$ (i.e., $f(x)<0$ for $x \in \Omega$ ) was also studied in the mentioned paper of S. Oruganti, J. Shi, R. Shivaji [26]. They used the bifurcation theory to prove (among other things) that under some additional assumptions the following hold for $\|f\|_{\infty}$ sufficiently small (see $[26$, Thm. 3.1, 3.3]):

(i) if $\lambda>\lambda_{1}$, there exists a positive solution $u_{1}$ of (5.1),

(ii) if $\lambda_{k}<\lambda<\lambda_{k+1}$, there exists a second solution $u_{2} \neq u_{1}$ of (5.1),

(iii) if $\lambda_{1}<\lambda<\lambda_{1}+\delta$ for some $\delta>0$ and $\lambda \neq \lambda_{k}$, the second solution $u_{2}$ of (5.1) is also positive.

Lately, P. Liu, J. Shi, Y. Wang [21] studied the exact multiplicity of solutions and obtained precise bifurcation diagrams for this problem on symmetric domains. Hence, Thm. 5.3 could contribute to this research and bring new insights via the critical point theory.

We justify the statement of Thm. 5.3 by an application of Thm. 1.1. Let us define the energy functional $F: W_{0}^{1,2}(\Omega) \rightarrow \mathbb{R}$ corresponding to (5.1) in the standard way as

$$
F(u)=\frac{1}{2} \int_{\Omega}|\nabla u(x)|^{2} \mathrm{~d} x-\frac{\lambda}{2} \int_{\Omega}|u(x)|^{2} \mathrm{~d} x+\frac{1}{p} \int_{\Omega}|u(x)|^{p} \mathrm{~d} x-\int_{\Omega} f(x) u(x) \mathrm{d} x .
$$

Applying the embedding $W_{0}^{1,2}(\Omega) \subset L^{p}(\Omega)$ one can rewrite the definition (5.4) into a shorter form as

$$
F(u)=\frac{1}{2}\|u\|^{2}-\frac{\lambda}{2}\|u\|_{2}^{2}+\frac{1}{p}\|u\|_{p}^{p}-\int_{\Omega} f(x) u(x) \mathrm{d} x .
$$

The following lemma can be proved in the same way as, e.g., D. G. Costa [11, Prop. 2.2.1].

Lemma 5.4. Let $f \in L^{2}(\Omega)$. Then for the functional $F$ given by (5.5) the following hold:

(i) $F \in C^{1}\left(W_{0}^{1,2}(\Omega), \mathbb{R}\right)$,

(ii) the Fréchet derivative of $F$ is given by

$$
\left\langle F^{\prime}(u), v\right\rangle=\int_{\Omega} \nabla u(x) \cdot \nabla v(x) \mathrm{d} x-\lambda \int_{\Omega} u(x) v(x) \mathrm{d} x+\int_{\Omega}|u(x)|^{p-2} u(x) v(x) \mathrm{d} x-\int_{\Omega} f(x) v(x) \mathrm{d} x,
$$

where $u, v \in W_{0}^{1,2}(\Omega)$,

(iii) $u \in W_{0}^{1,2}(\Omega)$ is a weak solution of (5.1) if and only if $u \in W_{0}^{1,2}(\Omega)$ is a critical point of $F$.

Consequently, it is satisfactory to show that $F$ has at least three critical points by Lem 5.4 (iii). Since $F \in$ $C^{1}\left(W_{0}^{1,2}(\Omega), \mathbb{R}\right)$ by Lem $5.4(i)$, it is enough to verify the remaining assumptions of Thm. 1.1.

Lemma 5.5. Let $f \in L^{2}(\Omega)$. Then for the functional $F$ given by (5.5) the following hold:

(i) $F$ is weakly coercive on $W_{0}^{1,2}(\Omega)$, i.e., $F(u) \rightarrow \infty$ for $\|u\| \rightarrow \infty$,

(ii) $F$ is bounded from below.

Proof. Since $2<p<\frac{2 N}{N-2}$, there is $W_{0}^{1,2}(\Omega) \subset L^{p}(\Omega) \subset L^{2}(\Omega)$ and the Hölder inequality yields

$$
\|u\|_{2} \leq(\operatorname{meas}(\Omega))^{\frac{p-2}{2 p}}\|u\|_{p} \quad \text { for all } \quad u \in W_{0}^{1,2}(\Omega) .
$$

Using (5.7) and the Hölder inequality again we obtain the following estimate

$$
\begin{aligned}
F(u) & =\frac{1}{2}\|u\|^{2}-\frac{\lambda}{2}\|u\|_{2}^{2}+\frac{1}{p}\|u\|_{p}^{p}-\int_{\Omega} f(x) u(x) \mathrm{d} x \\
& \geq \frac{1}{2}\|u\|^{2}-\frac{\lambda}{2}\|u\|_{2}^{2}+\frac{1}{p}(\operatorname{meas}(\Omega))^{\frac{2}{p-2}}\|u\|_{2}^{p}-\|f\|_{2}\|u\|_{2} .
\end{aligned}
$$

Let $\|u\| \rightarrow \infty$, then distinguishing between two possible cases we obtain:

(i) if $\|u\|_{2}$ is bounded, then $F(u) \rightarrow \infty$ thanks to $\|u\| \rightarrow \infty$,

(ii) if $\|u\|_{2} \rightarrow \infty$, then $F(u) \rightarrow \infty$ thanks to $2<p$.

Therefore, $F$ is weakly coercive. Moreover, one can continue in the estimate (5.8) to obtain

$$
F(u) \geq-\frac{\lambda}{2}\|u\|_{2}^{2}+\frac{1}{p}(\operatorname{meas}(\Omega))^{\frac{2}{p-2}}\|u\|_{2}^{p}-\|f\|_{2}\|u\|_{2}
$$

The right-hand side of (5.9) is a function of $\|u\|_{2}$ which is bounded from below for $\|u\|_{2} \geq 0$ (again thanks to $2<p)$. Hence, $F$ is bounded from below.

In the proof of the Palais-Smale condition we apply the following compactness argument which can be proved in the same way as P. H. Rabinowitz [31, Prop. B.35]. 
Lemma 5.6. Let $F$ be given by (5.5). If $\left\{u_{n}\right\} \subset W_{0}^{1,2}(\Omega)$ is a bounded sequence such that $F^{\prime}\left(u_{n}\right) \rightarrow o$, then $\left\{u_{n}\right\}$ has a convergent subsequence.

Consequently, the proof of the Palais-Smale condition is now restricted to the proof of boundedness of a PalaisSmale sequence. However, the boundedness follows immediately from the weak coercivity of $F$ which is guaranteed by Lem. 5.5 (i). Thus, the following lemma holds.

Lemma 5.7. Let $f \in L^{2}(\Omega)$. Then the functional $F$ given by (5.5) satisfies the Palais-Smale condition (PS).

Let us finally show that $F$ has the saddle geometry given by the assumption $(R)$. Let $\varphi_{i}, i \in \mathbb{N}$, be the normalized eigenfunctions corresponding to eigenvalues $\lambda_{i}$ which form an orthonormal basis of $W_{0}^{1,2}(\Omega)$ (see the Hilbert-Schmidt Theorem, e.g., P. Drábek, J. Milota [12, Thm. 2.2.16, Ex. 5.9.1]) denoted by

$$
\mathcal{B}=\left\{\varphi_{i}: i \in \mathbb{N}\right\}
$$

Recall that $\lambda_{k}<\lambda<\lambda_{k+1}$. We split $W_{0}^{1,2}(\Omega)$ into $Y \oplus Z$ with

$$
Y=\left\{\sum_{i=1}^{k} a_{i} \varphi_{i}: a_{i} \in \mathbb{R}, \varphi_{i} \in \mathcal{B}\right\}, \quad Z=\left\{\sum_{i=k+1}^{\infty} a_{i} \varphi_{i}: a_{i} \in \mathbb{R}, \varphi_{i} \in \mathcal{B}\right\}=Y^{\perp} .
$$

Lemma 5.8. Let $\lambda_{k}<\lambda<\lambda_{k+1}$. Then there exists $\mu>0$ such that the functional $F$ given by (5.5) satisfies ( $R$ ) with $Y, Z$ given by (5.10) for all $f \in L^{2}(\Omega)$ with $\|f\|_{2}<\mu$.

Proof. We proceed in two steps. Firstly, let $u \in Z$, i.e., $u=\sum_{i=k+1}^{\infty} a_{i} \varphi_{i}$ and by the Parseval equality $\|u\|^{2}=$ $\sum_{i=k+1}^{\infty} a_{i}^{2}$. Since $\varphi_{i}$ are the unit eigenfunctions of $-\Delta$ on $\Omega$ with the homogeneous Dirichlet boundary condition, i.e.,

and $\lambda<\lambda_{k+1}$, there is

$$
\lambda_{i} \int_{\Omega}\left|\varphi_{i}(x)\right|^{2} \mathrm{~d} x=\int_{\Omega}\left|\nabla \varphi_{i}(x)\right|^{2} \mathrm{~d} x=1 \quad \text { for all } \quad i \in \mathbb{N}
$$

$$
\int_{\Omega}|\nabla u(x)|^{2} \mathrm{~d} x-\lambda \int_{\Omega}|u(x)|^{2} \mathrm{~d} x=\sum_{i=k+1}^{\infty} a_{i}^{2}\left(1-\frac{\lambda}{\lambda_{i}}\right) \geq\left(1-\frac{\lambda}{\lambda_{k+1}}\right)\|u\|^{2} .
$$

Hence, we can estimate for $u \in Z$ applying (5.12) and the Hölder and Poincaré inequality

$$
\begin{aligned}
F(u) & \geq\left(1-\frac{\lambda}{\lambda_{k+1}}\right)\|u\|^{2}+\frac{1}{p}\|u\|_{p}^{p}-\int_{\Omega} f(x) u(x) \mathrm{d} x \\
& \geq\left(1-\frac{\lambda}{\lambda_{k+1}}\right)\|u\|^{2}-\lambda_{1}^{-\frac{1}{2}}\|f\|_{2}\|u\| \\
& \geq-\frac{\lambda_{1}\|f\|_{2}^{2}}{4\left(1-\frac{\lambda}{\lambda_{k+1}}\right)}
\end{aligned}
$$

The last inequality in (5.13) follows from the minimization of the quadratic function using $1-\frac{\lambda}{\lambda_{k+1}}>0$. There is also

$$
-\frac{\lambda_{1}\|f\|_{2}^{2}}{4\left(1-\frac{\lambda}{\lambda_{k+1}}\right)}<0
$$

Secondly, let $u \in Y$, i.e., $u=\sum_{i=1}^{k} a_{i} \varphi_{i}$ and $\|u\|^{2}=\sum_{i=1}^{k} a_{i}^{2}$. Applying (5.11) again and $\lambda_{k}<\lambda$ we obtain

$$
\int_{\Omega}|\nabla u(x)|^{2} \mathrm{~d} x-\lambda \int_{\Omega}|u(x)|^{2} \mathrm{~d} x=\sum_{i=1}^{k} a_{i}^{2}\left(1-\frac{\lambda}{\lambda_{i}}\right) \leq\left(1-\frac{\lambda}{\lambda_{k}}\right)\|u\|^{2} .
$$

Since $p<\frac{2 N}{N-2}$, the embedding $W_{0}^{1,2}(\Omega) \subset L^{p}(\Omega)$ is continuous (from the Sobolev Embedding Theorem, see, e.g., P. Drábek, J. Milota [12, Thm. 1.2.26]) and thus, there exists a constant $c>0$ such that (applying also (5.14) and the Hölder and Poincaré inequality)

$$
\begin{aligned}
F(u) & \leq\left(1-\frac{\lambda}{\lambda_{k}}\right)\|u\|^{2}+\frac{1}{p}\|u\|_{p}^{p}-\int_{\Omega} f(x) u(x) \mathrm{d} x \\
& \leq\left(1-\frac{\lambda}{\lambda_{k}}\right)\|u\|^{2}+\frac{c^{p}}{p}\|u\|^{p}+\lambda_{1}^{-\frac{1}{2}}\|f\|_{2}\|u\| .
\end{aligned}
$$

Therefore, we realize from (5.13) and (5.15) that $(R)$ is satisfied if we find $R>0$ such that the following holds for $u \in Y$ with $\|u\|=R$ 


$$
\left(1-\frac{\lambda}{\lambda_{k}}\right)\|u\|^{2}+\frac{c^{p}}{p}\|u\|^{p}+\lambda_{1}^{-\frac{1}{2}}\|f\|_{2}\|u\|<-\frac{\lambda_{1}\|f\|_{2}^{2}}{4\left(1-\frac{\lambda}{\lambda_{k+1}}\right)} .
$$

We rewrite (5.16) denoting $\|u\|=\rho$ into

$$
\left(1-\frac{\lambda}{\lambda_{k}}\right) \rho^{2}+\frac{c^{p}}{p} \rho^{p}<-\lambda_{1}^{-\frac{1}{2}}\|f\|_{2} \rho-\frac{\lambda_{1}\|f\|_{2}^{2}}{4\left(1-\frac{\lambda}{\lambda_{k+1}}\right)} .
$$

Define

$$
l(\rho)=\left(1-\frac{\lambda}{\lambda_{k}}\right) \rho^{2}+\frac{c^{p}}{p} \rho^{p}, \quad r(\rho)=-\lambda_{1}^{-\frac{1}{2}}\|f\|_{2} \rho-\frac{\lambda_{1}\|f\|_{2}^{2}}{4\left(1-\frac{\lambda}{\lambda_{k+1}}\right)} .
$$

One can see that the function $l$ is independent on $\|f\|_{2}$. Furthermore, $l$ has a strictly negative minimum at some $R>0$ thanks to $2<p$ and $\lambda_{k}<\lambda$. The function $r$ is a linear function which is nonpositive for $\rho>0$. However, $r(R) \rightarrow 0$ for $\|f\|_{2} \rightarrow 0$. Consequently, there is some $\mu>0$ such that

$$
l(R)<r(R) \text { for all }\|f\|_{2}<\mu .
$$

Thus, for all $f \in L^{2}(\Omega)$ such that $\|f\|_{2}<\mu$ and for all $u \in Y$ such that $\|u\|=R$ there is

$$
F(u)<-\frac{\lambda_{1}\|f\|_{2}^{2}}{4\left(1-\frac{\lambda}{\lambda_{k+1}}\right)} \leq \inf _{u \in Z} F(u),
$$

which verifies the assumption $(R)$.

Finally, we can conclude combining Lem. 5.4, Lem. 5.5 (ii), Lem. 5.7 and Lem. 5.8 together, that Thm. 5.3 is now justified by Thm. 1.1.

\section{Acknowledgments}

This publication was supported by the project LO1506 of the Czech Ministry of Education, Youth and Sports under the program NPU I.

The author is grateful to two anonymous reviewers for their careful proofreading and helpful suggestions. He also thanks his colleagues Pavel Drábek, Petr Stehlík, Radek Cibulka, Tomáš Kaiser, Hana Horníková and Vladimír Švígler for their valuable comments on this work.

\section{References}

[1] S. M. Allen, J. W. Cahn, A microscopic theory for antiphase boundary motion and its application to antiphase domain coarsening, Act. Met. 27 (1979), 1085-1095.

[2] A. Ambrosetti, P. H. Rabinowitz, Dual variational methods in critical point theory and applications, J. Funct. Anal. 14 (1973), 195-204.

[3] M. S. Berger, L. E. Fraenkel, On the Asymptotic Solution of a Nonlinear Dirichlet Problem, J. Math. Mech. 19 (1970), $553-585$.

[4] G. Bonanno, A characterization of the mountain pass geometry for functionals bounded from below, Diff. Integral Equ. 25 (2012), 1135-1142.

[5] G. Bonanno, Relations between the mountain pass theorem and local minima, Adv. Nonlin. Anal. 1 (2012), 205-220.

[6] H. Brezis, L. Nirenberg, Remarks on Finding Critical Points, Commun. Pur. Appl. Math. 44 (1991), 939-963.

[7] A. Castro, A. C. Lazer, Critical point theory and the number of solutions of a nonlinear Dirichlet problem, Ann. Mat. Pur. Appl. 120 (1979), 113-137.

[8] A. Castro, R. Shivaji, Nonnegative solutions for a class of nonpositone problems, Proc. Roy. Soc. Edin. 108(A) (1988), 291-302.

[9] A. Castro, C. Maya, R. Shivaji, Nonlinear eigenvalue problems with semipositone structure, Electronic J. Diff. Eqns. Conf. 5 (2000), 33-49.

[10] D. C. Clark, A variant of Ljusternik-Schnirelmann theory, Indiana U. Math. J. 22 (1972), 65-74.

[11] D. G. Costa, An Invitation to Variational Methods in Differential Equations, Birkhäuser, Boston, 2007.

[12] P. Drábek, J. Milota, Methods of Nonlinear Analysis, Birkhäuser, Basel, 2013.

[13] I. Ekeland, On the variational principle, J. Math. Anal. Appl. 47 (1974), 324-353.

[14] R. A. Fisher, The wave of advance of advantageous genes, Ann. Eugenics 7 (1937), 353-369. 
[15] N. Ghoussoub, D. Preiss, A general mountain pass principle for locating and classifying critical points, Ann. I. H. Poincaré An. 6 (1989), 321-330.

[16] D. Henry, Geometric theory of semilinear parabolic equations, Springer-Verlag, Berlin, 1981.

[17] D. Kandilakis, N. C. Kourogenis, N. S. Papageorgiou, Two Nontrivial Critical Points for Nonsmooth Functionals via Local Linking and Applications, J. Global Optim. 34 (2006), 219-244.

[18] A. Kolmogorov, I. Petrovskii, N. Piskunov, Study of a Diffusion Equation That Is Related to the Growth of a Quality of Matter and Its Application to a Biological Problem, Mosc. U. Math. Bull. 1 (1937), 1-26.

[19] A. C. Lazer, E. M. Landesman, D. R. Meyers, On Saddle Point Problems in the Calculus of Variations, the Ritz Algorithm, and Monotone Convergence, J. Math. Anal. Appl. 52 (1975), 594-614.

[20] S. Li, M. Willem, Applications of Local Linking to Critical Point Theory, J. Math. Anal. Appl. 189 (1995), 6-32.

[21] P. Liu, J. Shi, Y. Wang, Exact multiplicity of solutions to perturbed logistic type equations on a symmetric domain, Sci. China Ser. A 51 (2008), 1753-1762.

[22] Z. Liu, J. Sun, Invariant Sets of Descending Flow in Critical Point Theory with Applications to Nonlinear Differential Equations, J. Differ. Equ. 172 (2001), 257-299.

[23] R. Manásevich, A Min Max Theorem, J. Math. Anal. Appl. 90 (1982), 64-71.

[24] J. Mawhin, M. Willem, Critical point theory and Hamiltonian systems, Springer-Verlaq, New York, 1989.

[25] J. D. Murray, Mathematical biology, Springer, New York, 2002.

[26] S. Oruganti, J. Shi, R. Shivaji, Diffusive logistic equation with constant effort harvesting, I: Steady States, Trans. Amer. Math. Soc. 354 (2002), 3601-3619.

[27] N. C. Owen, J. Rubinstein, P. Sternberg, Minimizers and gradient flows for singularly perturbed bi-stable potentials with a Dirichlet condition, Proc. Roy. Soc. Lon. Ser. A 429 (1990), 505-532.

[28] R. S. Palais, Critical point theory and the minimax principle, Proc. Symp. Pure Math. XV, American Mathematical Society, Providence, 1970, 185-212.

[29] P. Pucci, J. Serrin, A mountain pass theorem, J. Differ. Equ. 60 (1985), 142-149.

[30] P. H. Rabinowitz, A Note on Topological Degree for Potential Operators, J. Math. Anal. Appl. 51 (1975), $483-492$.

[31] P. H. Rabinowitz, Minimax Methods in Critical Point Theory with Applications to Differential Equations, American Mathematical Society, Providence, 1986.

[32] P. H. Rabinowitz, Some minimax theorems and applications to nonlinear partial differential equations, Nonlinear Analysis: A collection of papers in honor of Erich Röthe, Academic Press, New York, 1978, 161-177.

[33] B. Ricceri, On a three critical points theorem, Arch. Math. 75 (2000), 220-226.

[34] X. Wu, A new critical point theorem for locally Lipschitz functionals with applications to differential equations, Nonlin. Anal. 66 (2007), 624-638. 